\title{
THE COLORED DISCONNECTION NUMBERS OF CELLULAR AND GRID NETWORKS
}

\author{
Xuqing Bai ${ }^{1}$ and Xueliang $\mathrm{Li}^{2}$ and \\ Yindi Weng ${ }^{3}$ \\ Center for Combinatorics and LPMC Nankai University, Tianjin, China
}

\begin{abstract}
Let $G$ be a nontrivial link-colored connected network. A link-cut $R$ of $G$ is called a rainbow link-cut if no two of its links are colored the same. A link-colored network $G$ is rainbow disconnected if for every two nodes $u$ and $v$ of $G$, there exists a $u$-v rainbow link-cut separating them. Such a link coloring is called a rainbow disconnection coloring of $G$. For a connected network $G$, the rainbow disconnection number of $G$, denoted by $r d(G)$, is defined as the smallest number of colors that are needed in order to make G rainbow disconnected. Similarly, there are some other new concepts of network colorings, such as proper disconnection coloring, monochromatic disconnection coloring and rainbow node-disconnection coloring.
\end{abstract}

In this paper, we obtain the exact values of the rainbow (node-)disconnection numbers, proper and monochromatic disconnection numbers of cellular networks and grid networks, respectively.

\section{KEYWORDS}

link- (node-)coloring, connectivity, rainbow link- (node-)cut, (strong) rainbow (node-)disconnection numbers, proper and monochromatic disconnection numbers, cellular network, grid network

\section{INTRODUCTION}

All networks (also called graphs) considered in this paper are simple, finite and undirected. Let $G=(V$ $(G), E(G)$ ) be a nontrivial connected network with node set $V(G)$ and link set $E(G)$. The order of $G$ is denoted by $n=|V(G)|$. For a node $v \in V(G)$, the open neighborhood of $v$ is the set $N(v)=\{u \in V$ $(G) \mid u v \in E(G)\}$ and $d(v)=|N(v)|$ is the degree of $v$, and the closed neighborhood of $v$ is the set $N[v]$ $=N(v) \cup\{v\}$. The minimum and maximum degree of $G$ are denoted by $\delta(G)$ and $\Delta(G)$, respectively. Denote by $P_{n}$ a path on $n$ nodes. For a subset $S$ of $V(G)$, we use $G[S]$ to denote the subnetwork of $G$ induced by $S$. Let $V_{1}, V_{2}$ be two disjoint node subsets of $G$. We denote the set of links between $V_{1}$ and $V_{2}$ in $G$ by $E\left(V_{1}, V_{2}\right)$. We follow [7] for network theoretical notation and terminology not defined here.

The concept of rainbow connection coloring was introduced by Chartrand et al. [10] in 2008. A rainbow path is a path whose links are colored pairwise differently. A link-coloring of a network $G$ is a rainbow connection coloring if any two nodes of $G$ are connected by a rainbow path. The rainbow connection number of a connected network $G$, denoted by $\operatorname{rc}(G)$, is the minimum number of colors so that $G$ has a rainbow connection coloring. Rainbow node-connection was proposed by Krivelevich and Yuster [12] in 2010. For more details about the rainbow (node-)connection, we refer to [13] and survey papers $[14,16]$ and book [15].

As we know that there are two ways to study the connectivity of a network, one way is by using paths and the other is by using cuts. The above rainbow connection and rainbow node-connection use paths. 
So, it is natural to consider the rainbow link-cuts and rainbow node-cuts for the colored connectivity in colored networks.

In [8], Chartrand et al. first studied the rainbow link-cuts by introducing the concept of rainbow disconnection of networks, and later produced some other new concepts of colored disconnection colorings, such as proper disconnection coloring and monochromatic disconnection coloring. Let $G$ be a nontrivial connected and link-colored network. A link-cut of $G$ is a set $R$ of links of $G$ such that $G-$ $R$ is disconnected. If all (adjacent, no two) links in $R$ have different colors, then $R$ is called a rainbow (proper, monochromatic) link-cut. Let $u$ and $v$ be two nodes. A rainbow (proper, monochromatic) linkcut $R$ is called a $u$-v rainbow (proper, monochromatic) link-cut if the nodes $u$ and $v$ belong to different components of $G-R$. A link-coloring of $G$ is called a rainbow (proper, monochromatic) disconnection coloring (abbreviated as rd-coloring, pd-coloring and md-coloring) if for every two distinct nodes $u$ and $v$ of $G$, there exists a $u-v$ rainbow (proper, monochromatic) link-cut in $G$, separating them. The rainbow (proper) disconnection number (abbreviated as $\mathrm{rd}(\mathrm{pd})$-number) $\operatorname{rd}(G)(\operatorname{pd}(G))$ of $G$ is the minimum number of colors required by a rainbow (proper) disconnection coloring of $G$. The monochromatic disconnection number (abbreviated as md-number) $\operatorname{md}(G)$ of $G$ is the maximum number of colors required by a monochromatic disconnection coloring of $G$.

In fact, the rainbow disconnection number has the following application background. In some illegal commodity transactions, we hope to stop the transaction in time and send out a signal (a certain frequency). On the one hand, we need to block all the roads between the two cities and identify the interception locations based on different signals; on the other hand, we want to use as few frequencies as possible in order to reduce costs. Therefore, we want to know what is the minimum frequency required to meet the above requirements? Treat each city as a node. If there is a road between two cities, we add a link between the two nodes, and use $G$ to denote the resulting network. Give a linkcoloring for $G$, where the color on the link corresponds to the frequency of the road. Therefore, the above problem is equivalent to calculating the rainbow disconnection number of the network $G$.

In order to study the rainbow node-cut, we introduce the concept of rainbow node-disconnection number in this paper. For a connected and node-colored network $G$, let $x$ and $y$ be two nodes of $G$. If $x$ and $y$ are nonadjacent, then an $x-y$ node-cut is a subset $S$ of $V(G)$ such that $x$ and $y$ belong to different components of $G-S$. If $x$ and $y$ are adjacent, then an $x-y$ node-cut is a subset $S$ of $V(G)$ such that $x$ and $y$ belong to different components of $(G-x y)-S$. A node subset $S$ of $G$ is rainbow if no two nodes of $S$ have the same color. An $x-y$ rainbow node-cut is an $x-y$ node-cut $S$ such that if $x$ and $y$ are nonadjacent, then $S$ is rainbow; if $x$ and $y$ are adjacent, then $S+x$ or $S+y$ is rainbow.

A node-colored network $G$ is called rainbow node-disconnected if for any two nodes $x$ and $y$ of $G$, there exists an $x-y$ rainbow node-cut. In this case, the node-coloring $c$ is called a rainbow nodedisconnection coloring of $G$. For a connected network $G$, the rainbow node-disconnection number of $G$, denoted by $\operatorname{rnd}(G)$, is the minimum number of colors that are needed to make $G$ rainbow nodedisconnected. A rainbow node-disconnection coloring with $\operatorname{rnd}(G)$ colors is called an rnd-coloring of $G$.

Remember that in the Menger's Theorem, only minimum link-cuts play a role, however, in the definition of rd-colorings we only requested the existence of a $u$-v link-cut between nodes $u$ and $v$, which could be any link-cut (large or small are both OK). This may cause the failure of a colored version of such a nice Min- Max result. In order to overcome this problem, we introduced the concept of strong rainbow disconnection in networks in [5], with a hope to set up the colored version of the socalled Max-Flow Min-Cut Theorem.

A link-colored network $G$ is called strong rainbow disconnected if for every two distinct nodes $u$ and $v$ of $G$, there exists a both rainbow and minimum $u$-v link-cut (rainbow minimum $u$-v link-cut for short) in $G$. Such a link-coloring is called a strong rainbow disconnection coloring (abbreviated as srd- 
coloring) of $G$. For a connected network $G$, similarly, the strong rainbow disconnection number (abbreviated as srd-number) of $G$, denoted by $\operatorname{srd}(\mathrm{G})$, is the minimum number of colors required to make $\mathrm{G}$ strong rainbow disconnected. We call the colored (dis)connection numbers the global chromatic numbers, and the classic or traditional chromatic numbers the local chromatic numbers [6].

The rapid development of computer networks and communication technology, and the rise and wide application of internet technology have strongly promoted the development of commercial applications and scientific applications in the network environment, such as grid networks $[1,2,9]$ and cellular networks $[18,19]$. The cellular network is a mobile communication hardware architecture that divides the service of mobile phones into small regular hexagonal sub-areas, and each cell has a base station, forming a structure that resembles a "cellular" structure. Therefore, this mobile communication method is called cellular mobile communication method, and its structure can save equipment construction costs. The grid networks were developed to support large-scale scientific collaborative work.

Based on the importance of cellular networks and grid networks, it is natural to consider the disconnection colorings of them.

Consider a (planar, infinite) lattice of congruent regular hexagons (quadrangle) and a cycle $C$ on it. Then the part of the hexagonal (quadrangle) lattice which lies in the interior of $C$ and the cycle $C$ itself, forms a cellular networks (grid networks) $G$ [11]. We call the $C$ the boundary of the network $G$. Denote by $E(G)-C$ the inner links of $G$. Obviously, the cellular networks and grid networks are 2connected.

This paper is organized as follows. In Section 2, we obtain the (strong) rainbow disconnection numbers of cellular networks and grid networks. In Section 3, we give the rainbow node-disconnection numbers of cellular networks and grid networks. In Section 4, we present the proper and monochromatic disconnection numbers of cellular networks and grid networks.

\section{THEIR RD-NUMBERS AND SRD-NUMBERS}

For two distinct nodes $u$ and $v$ of $G$, let $\lambda_{G}(u, v)$ (or simply $\lambda(u, v)$ when the network $G$ is clear from the context) denote the minimum number of links in a link-cut $F$ such that $u$ and $v$ lie in different components of $G-F$. The minimum cardinality of a link-cut of $G$ is the link-connectivity of $G$, denoted by $\lambda(G)$.

Lemma 2.1 [8] If G is a nontrivial connected network, then

$$
\lambda(G) \leq \lambda^{+}(G) \leq r d(G) \leq \chi^{\prime}(G) \leq \Delta(G)+1,
$$

where the upper link-connectivity $\lambda^{+}(G)$ is defined by $\lambda^{+}(G)=\max \{\lambda(u, v): u, v \in V(G)\}$.

Lemma 2.2 [8] Let $G$ be a nontrivial connected network. Then $r d(G)=2$ if and only if each block of $G$ is either $K_{2}$ or a cycle and at least one block of $G$ is a cycle.

Theorem 2.3 Let $G$ be a cellular network with the number of hexagons $h$. Then

$$
\operatorname{rd}(G)= \begin{cases}2, & \text { if } h=1, \\ 3, & \text { if } h \geq 2\end{cases}
$$

Proof. If $h=1$, then $G=C_{6}$, so $\operatorname{rd}(G)=2$ by Lemma 2.2. If $h \geq 2$, there exist two nodes $u$, $v$ of $G$ 
satisfying $\lambda(u, v)=3$. Moreover, we have $\chi^{\prime}(G)=\Delta(G)=3$ since $G$ is a bipartite network. Hence, we get $\operatorname{rd}(G)=3$ by Lemma 2.1 .

Theorem 2.4 Let G be a grid network. Then (see Figure 1)

$$
\operatorname{rd}(G)= \begin{cases}2, & \text { if } G=G_{1}, \\ 3, & \text { if } H \subseteq G \text { and } H \cong G_{2}, \text { but no } H \subseteq G \text { and } H \cong G_{3}, \\ 4, & \text { if } H \subseteq G \text { and } H \cong G_{3} .\end{cases}
$$

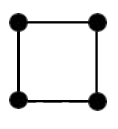

$\mathrm{G}_{1}$

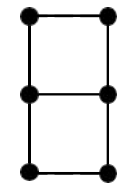

$\mathrm{G}_{2}$

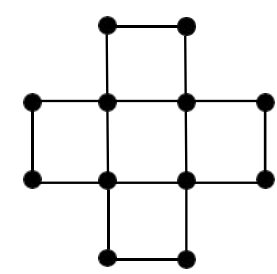

$\mathrm{G}_{3}$

Figure 1: Grid networks in Theorem 2.4.

Proof. If $G=G_{1}$, then $\operatorname{rd}(G)=2$. If $G_{3} \subseteq G$, then $\lambda^{+}(G)=4$. Moreover, since $G$ is a bipartite network we have $\operatorname{rd}(G) \leq \chi^{\prime}(G)=\Delta(G)=4$. Hence, $\operatorname{rd}(G)=4$.

Suppose that $G$ has a subnetwork that is isomorphic to $G_{2}$, but no subnetwork that is isomorphic to $G_{3}$. Then we get $\operatorname{rd}(G) \geq 3$ by Lemma 2.1 since $\lambda^{+}(G)=3$. It remains to prove that there exists an rdcoloring of $\mathrm{G}$ using 3 colors. First, we give two observations.

1. For any two nodes $x$ and $y$ of $G$ with $d(x)=d(y)=4$, if there has no a parallel 2 (3)-link-cut between $x$ and $y$, then we can find a 3-link-cut $C(x, y)$ of $x, y$ in $G$ (see Figure 2).

2. For such two different 3-link-cuts in $G$, they have at most one common link in $G$, which ensures that there exists a coloring using colors [3] so that each 3-link-cut (like $C(x, y)$ in Figure 2) is rainbow.

We now divide these link-cuts into some families of link-cut: if two link-cuts belong to the same family, then one can find the other link-cut by link transitivity. Let $G^{*}$ be the network obtained by deleting all such 3-link-cuts (like $C(x, y)$ in Figure 2) of $G$. Note that each nontrivial block of $G^{*}$ is a subnetwork of $G_{3, i}(i \geq 3)$. We first assign a coloring $c_{0}$ for one component of $G^{*}$, say $H_{0}$, using colors [3] so that each set of links incident with a node of degree less than 4 and parallel 2 (3)-link-cuts in $G$ are rainbow. Then, we color a family of link-cuts connected to the network $H_{0}$ so that each link-cut is a rainbow and each node is proper except for the nodes of degree 4 in $G$, and use $H_{1}$ to denote the new colored network. Furthermore, we colored other component of $G^{*}$ connected with network $H_{1}$ and ensure that each node of $H_{1}$ is proper except the nodes of degree 4 in $G$ and all parallel 2 (3)-link-cuts in $G$ are rainbow. Repeatedly, we extent the coloring $c_{0}$ to a coloring $c$ of $G$ using colors [3] so that each parallel 2 (3)-link-cut and each set of links incident with a node of degree less than 4 in $G$ is rainbow.

Now we can verify that the $c$ is an rd-coloring of $G$. For any two nodes $u$, $v$ of $G$, if there exists a node 


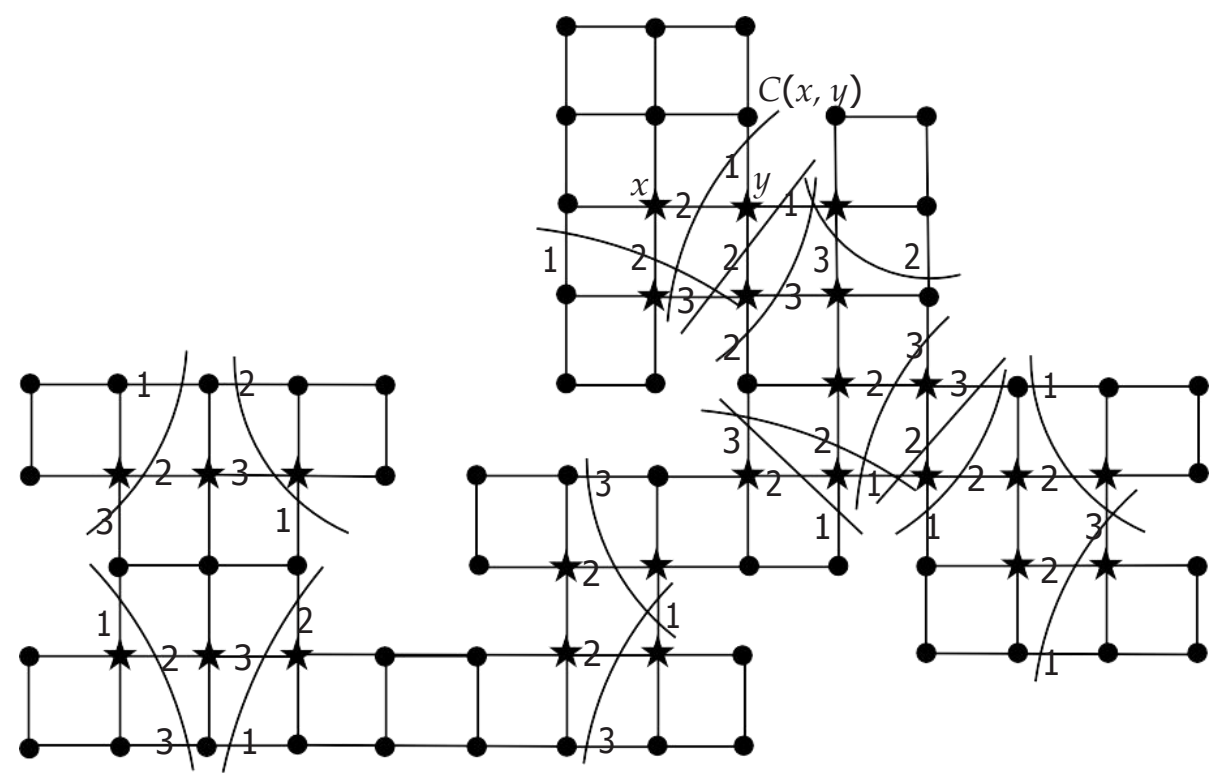

Figure 2: A network used in the proof of Theorem 2.4.

with degree less than 4 , without loss of generality, say $u$, then the set $E_{u}$ of links incident with node $u$ is a $u-v$ rainbow link-cut. If $d(u)=d(v)=4$ and there has a parallel $u-v 2$ (3)-link-cut, then it is a $u-v$ rainbow link-cut. If $d(u)=d(v)=4$ and there has no parallel $u$-v 2 (3)-link-cut, then the $C(u, v)$ (like $C(x, y)$ in Figure 2) in network $G$ is a $u$-v rainbow link-cut in $G$.

Furthermore, we study the strong rainbow disconnection numbers of cellular networks.

A trivial link-cut $S$ of $G$ is a link-cut incident with a node.

Lemma 2.5 [5] If $G$ is a connected network with link-connectivity $\lambda(G)$, upper link-connectivity $\lambda^{+}(G)$ and number $e(G)$ of links, then

$$
\lambda(G) \leq \lambda^{+}(G) \leq \operatorname{rd}(G) \leq \operatorname{srd}(G) \leq e(G) .
$$

Lemma 2.6 [17] A 3-connected cubic plane network $G$ is 4-face-colorable if and only it is 3-link colorable, i.e., $\chi^{\prime}(G)=3$.

Lemma 2.7 [4] A cube network $G$ is 3-connected if and only if $G$ is 3-link-connected.

Lemma 2.8 [5] Let $G$ be a nontrivial connected network. Then $\operatorname{srd}(G)=2$ if and only if $r d(G)=2$.

Theorem 2.9 Let $G$ be a cellular network with the number of hexagons $h$. Then

$$
\operatorname{srd}(G)= \begin{cases}2, & \text { if } h=1, \\ 3, & \text { if } h \geq 2 .\end{cases}
$$

Proof. If $h=1$, then $G=C_{6}$. By Lemmas 2.2 and 2.8, we have $\operatorname{srd}(G)=2$. If $h \geq 2$, there exist two nodes $u, v$ of $G$ satisfying $\lambda(u, v)=3$, so $\operatorname{srd}(G) \geq 3$ by Lemma 2.5. Now we define two operations $o$ and $O$ as follows. 


$$
\begin{gathered}
o(\{G\})= \begin{cases}\left\{G / V\left(C_{1}\right), G / V\left(C_{2}\right)\right\}, & \text { if } G \text { has a nontrivial 2-link-cut } S \\
& \text { and } G \backslash S=C_{1} \cup C_{2}, \\
\{G\}, & \text { otherwise. }\end{cases} \\
\qquad O\left(\left\{G_{1}, G_{2}, \cdots, G_{p}\right\}\right)=\cup_{i=1}^{p} o\left(\left\{G_{i}\right\}\right) .
\end{gathered}
$$

Since the network is split into two pieces when we do the operation, then the operation cannot last endlessly. Hence, there exists a integer $r$ such that $O^{r}(\{G\})=O^{r+1}(\{G\})$. Finally, we get a finite sequence of link-colored cubic networks $\mathrm{H}=\left\{H_{1}, H_{2}, \cdots, H_{q}\right\}$, where $q$ is a positive integer. Note that the operation does not appear multilinks, and each network of $\left\{H_{1}, H_{2}, \cdots, H_{q}\right\}$ is planar. For each planar network $H \in \mathrm{H}$, we can construct a 3-link-connected 3-regular planar network $H^{\prime}$. By the above operation, we know that each 2-link-cut in $H$ is trivial and lies on the boundary of network $H$. Let $h$ be the number of nodes with degree 2. Use $h$ to denote the number of trivial 2-link-cuts in network $H$ and give all nodes with degree 2 a clockwise label using $\left\{v_{i}: i \in[h]\right\}$. If $h \equiv 0(\bmod 3)$, then we add $h / 3$ nodes, and make each node connect with 3 adjacent 2-degree nodes in $H$ (starting from the node with degree 2 labeled 1, connect the links in turn clockwise, the same below); if $h \equiv 1$ $(\bmod 3)$, we add $\lfloor h / 3\rfloor-1$ nodes, and make each node connect to the 3 adjacent nodes with degree 2 in $H$. For the remaining 4 nodes with degree 2, we add two links $v_{h-3} v_{h-2}$ and $v_{h-1} v_{h}$; if $h \equiv 2(\bmod 3)$, we add $[h / 3\rfloor$ nodes, and make each node connect to the 3 adjacent nodes with degree 2 in $H$, and then add a link between the remaining two nodes with degree 2 . It is easy to verify that the network $H^{\prime}$ is a 3link-connected 3-regular plane network. By Lemmas 2.6 and 2.7, it implies that $H^{\prime}$ is 3-link-colorable. Then each network $H$ is 3-link-colorable, and we use color set [3] to assign a proper link-coloring to each network in $\mathrm{H}$. Then we perform the inverse operation of the shrinking operation. Assume that $F_{1}$ and $F_{2}$ are two proper link-colored networks obtained by shrinking the non-trivial 2-link-cut $\left\{e_{1}, e_{2}\right\}$ of network $F$, and let $c_{1}$ and $c_{2}$ be colorings of networks $F_{1}$ and $F_{2}$ using colors [3], respectively. Obviously, $c_{1}\left(e_{1}\right) \neq c_{1}\left(e_{2}\right)$ and $c_{2}\left(e_{1}\right) \neq c_{2}\left(e_{2}\right)$. Now we exchange the colors $c_{1}\left(e_{1}\right)$ and $c_{2}\left(e_{1}\right)$, and colors $c_{1}\left(e_{2}\right)$ and $c_{2}\left(e_{2}\right)$ in $F_{1}$ such that the new coloring $c_{1}^{\prime}$ of $F_{1}$ satisfies $c_{1}^{\prime}\left(e_{1}\right)=c_{2}\left(e_{1}\right)$ and $c_{1}^{\prime}\left(e_{2}\right)=c_{2}\left(e_{2}\right)$. Obviously, $c_{1}^{\prime}$ is still a proper link-coloring of the network $F_{1}$ using the color set [3]. Then we can get a link-coloring $c_{0}$ of network F: let $c_{0}(e)=c_{1}^{\prime}(\mathrm{e})$, if e $\in F_{1}$; let $c_{0}(e)=c_{2}(e)$, if $e \in F_{2}$. Obviously, the $c_{0}$ is a proper link-coloring of network $F$. Continue to do this, and finally we get a proper link-coloring $c$ of the network $G$ using the color set [3].

Now we verify that the link-coloring $c$ of $G$ is a strong rainbow disconnection coloring of the network $G$. Let $u$ and $v$ be two nodes of $G$, and assume that $d(u) \leq d(v)$. If $d(u)=2$, then the link set $E_{u}$ is a minimum u-v link-cut of $G$ and rainbow, so the link set $E_{u}$ is a rainbow minimum u-v link-cut of $G$; if $d(u)=d(v)=3$ and $\lambda(u, v)=3$, then the link set $E_{u}$ is a minimum $u$-v link-cut of $G$ and rainbow, so the link set $E_{u}$ is a rainbow minimum $u$-v link-cut of $G$; if $d(u)=d(v)=3$ and $\lambda(u, v)=2$. By the contraction operation, we get that $u$ and $v$ belong to different connected components in $H$ (otherwise, suppose that both $u$ and $v$ belong to a connected component $H$ of $\mathrm{H}$. Since $\lambda(u, v)=2$, and the shrinking operation does not change the link connectivity of $u, v$, there is still a nontrivial 2-link-cut between $u$ and $v$. This is a contradiction with our operation). Therefore, there exists a rainbow 2-linkcut $C(u, v)$ between $u$ and $v$ by the process of operation and coloring, and the $C(u, v)$ is a rainbow minimum u-v link-cut of $G$. Hence, $\operatorname{srd}(G) \leq 3$.

Moreover, we conjecture that the strong rainbow disconnection numbers of grid networks are equal to the rainbow disconnection numbers of grid networks.

Conjecture 2.10 Let G be a grid network (see Figure 1). Then 


$$
\operatorname{srd}(G)= \begin{cases}2, & \text { if } G=G_{1}, \\ 3, & \text { if } H \subseteq G \text { and } H \cong G_{2}, \text { but no } H \subseteq G \text { and } H \cong G_{3}, \\ 4, & \text { if } H \subseteq G \text { and } H \cong G_{3} .\end{cases}
$$

\section{THEIR RND-NUMBERS}

Next, we study the node-version of rainbow disconnection coloring.

Lemma 3.1 [3] If $C_{n}$ is a cycle of order $n \geq 3$, then $\operatorname{rnd}\left(C_{n}\right)=2$.

Lemma 3.2 [3] If $G$ is a nontrivial connected network and $H$ is a connected subnetwork of $G$, then $\operatorname{rnd}(H) \leq \operatorname{rnd}(G)$.

Lemma 3.3 [3] Let $G$ be a nontrivial connected network of order $n$. Then $\kappa(G) \leq \kappa^{+}(G) \leq \operatorname{rnd}(G) \leq n$.

Theorem 3.4 Let $G$ be a cellular network with the number of hexagons $h$. Then

$$
\operatorname{rnd}(G)= \begin{cases}2, & \text { if } h=1, \\ 3, & \text { if } h \geq 2 .\end{cases}
$$

Proof. If $h=1$, then we have $\operatorname{rnd}(G)=2$ by Lemma 3.1. If $h \geq 2$, we select the common link of some two hexagons, say $v_{1} v_{2}$. We have $\operatorname{rnd}(G) \geq \kappa_{G}\left(v_{1}, v_{2}\right) \geq 3$. For the nodes of $G$, assign column numbers according to the order in which they appear from left to right in the lattice shown in the figure 3 . For example, the nodes in the same column which appear first are labeled column 1 . Now we give a nodecoloring $c$ of $G$ using three colors. For the nodes in the column $j$ of network $G$, if $j \equiv 1(\bmod 3)$, then color them by 1 ; if $j \equiv 2(\bmod 3)$, then color them by 2 ; if $j \equiv 0(\bmod 3)$, then color them by 3 . Let $v$ be any node of network $G$. Assume that $v$ is in the column $i$ of $G$. If $d_{G}(v)=2$, then the neighbors of $v$ are in columns $i-1, i+1$ or $i, i+1$ or $i-1, i$. Since the column labels of the neighbors are different modulo 3, we have $N_{G}(v)$ is rainbow. If $d_{G}(v)=3$, then the neighbors of $v$ are in columns $i-1, i, i+1$, respectively. Since $i-1, i, i+1$ are pairwise different modulo 3 , we have that $N_{G}(v)$ is rainbow.

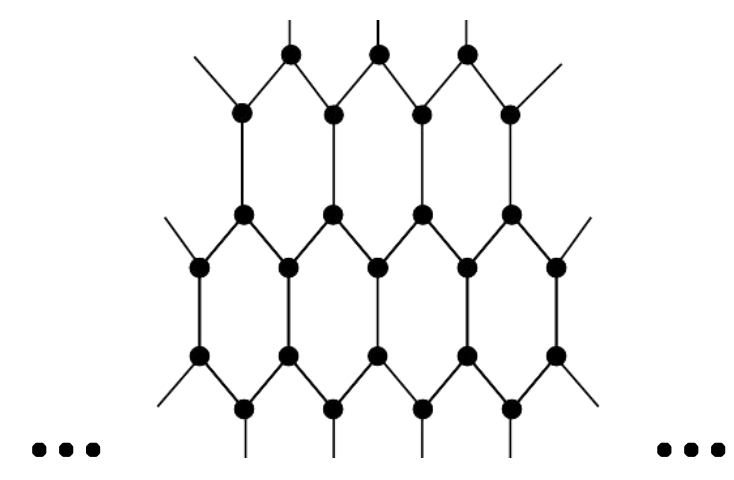

Figure 3: A (planar, infinite) lattice of congruent regular hexagons.

Let $x$ and $y$ be two nodes of network $G$. If $x, y$ are adjacent, then $N_{G}(x) \backslash\{y\}$ is an $x-y$ rainbow node-cut. If $x, y$ are nonadjacent, then $N_{G}(x)$ is an $x-y$ rainbow node-cut. So $c$ is a rainbow node-disconnection coloring of network $G$. We obtain $\operatorname{rnd}(G) \leq 3$. 
The Cartesian product $G$ 口 $H$ of two internal disjoint networks $G$ and $H$ is the network with node set $V(G) \times V(H)$, where $(u, v)$ is adjacent to $(w, x)$ in $G$ 口 $H$ if and only if either $u=w$ and $v x \in E(H)$ or $u w \in E(G)$ and $v=x$. The $m \times n$ grid network $G_{m, n}=P_{m}$ 口 $P_{n}$ consists of $m$ horizontal paths $P_{n}$ and $n$ vertical paths $P_{m}$.

Lemma 3.5 For $n \geq 3, \operatorname{rnd}\left(G_{3, n}\right)=3$.

Proof. Define a node-coloring $c: V\left(G_{3, n}\right) \rightarrow[3]$ of $G_{3, n}$. Let $c\left(x_{1, j}\right)=1$ for $j \equiv 1,2(\bmod 4)$ and $c\left(x_{1, j}\right)=$ 2 for $j \equiv 0,3(\bmod 4)$. We color the second row using color 3 . Let $c\left(x_{3, j}\right)=2$ for $j \equiv 1,2(\bmod 4)$ and $c\left(x_{3, j}\right)=1$ for $j \equiv 0,3(\bmod 4)$. We show that $c$ is a rainbow node-disconnection coloring of $G_{3, n}$. Let $x_{p, q}$ and $x_{s, \ell}$ be two nodes of network $G_{3, n}$, where $p \leq s$.

If $p=1$, then $N_{G_{3, n}}\left(x_{p, q}\right)$ is rainbow. So when $x_{p, q}$ and $x_{s, l}$ are nonadjacent, $N_{G_{3, n}}\left(x_{p, q}\right)$ is an $x_{p, q^{-}} x_{s, \ell}$ rainbow node-cut; when $x_{p, q}$ and $x_{s, \ell}$ are adjacent, $N_{G_{3, n}}\left(x_{p, q}\right) \backslash\left\{x_{s, \ell}\right\}$ is an $x_{p, q}-x_{s, \ell}$ rainbow node-cut. If $s=$ 3 , then $N_{G_{3, n}}\left(x_{s, \ell}\right)$ is rainbow. Similarly, there is a rainbow node-cut between $x_{p, q}$ and $x_{s, \ell}$.

Now consider $p=s=2$. Suppose that $q<\ell$. If $x_{p, q}$ and $x_{s, \ell}$ are nonadjacent, $\left\{x_{p-1, q}, x_{p, q+1}, x_{p+1, q}\right\}$ is an $x_{p, q}-x_{s, \ell}$ rainbow node-cut. If $x_{p, q}$ and $x_{s, \ell}$ are adjacent, $\left\{x_{p-1, q}, x_{p+1, q}\right\}$ is an $x_{p, q}-x_{s, \ell}$ rainbow node-cut.

So we have $\operatorname{rnd}\left(G_{3, n}\right) \leq 3$. Since $\kappa\left(x_{1,2}, x_{2,2}\right)=3$, we have $\operatorname{rnd}(G) \geq \kappa\left(x_{1,2}, x_{2,2}\right)=3$ by Lemma 3.3.

Lemma 3.6 For $4 \leq m \leq n, \operatorname{rnd}\left(G_{m, n}\right)=4$.

Proof. Define a node-coloring $c$ of $G_{m, n}: V\left(G_{m, n}\right) \rightarrow \mathrm{Z}_{4}$. Let $c\left(x_{i, 1}\right)=i(\bmod 4), c\left(x_{i, 2}\right)=c\left(x_{i, 3}\right)=i+2$ $(\bmod 4)$ and $c\left(x_{i, 4}\right)=i(\bmod 4)$. Other remaining columns repeat the coloring of first four columns.

Let $u$ be a node of $G_{m, n}$ and $N_{r}(u)\left(N_{c}(u)\right)$ denote the neighbors of $u$ in the same row (column). Assume that $c(u)=a$. If $\left|N_{r}(u)\right|=2$, then two nodes of $N_{r}(u)$ are assigned $a$ and $a+2$ respectively; if $\left|N_{r}(u)\right|=1$, then it is assigned $a$ or $a+2$. If $\left|N_{c}(u)\right|=2$, then two nodes of $N_{c}(u)$ are assigned $a-1$ and $a+1$ respectively; if $\left|N_{r}(u)\right|=1$, then it is assigned $a-1$ or $a+1$. Thus, $N_{G_{m, n}}(u)$ is rainbow.

For any two nonadjacent nodes $x$ and $y$ of $G_{m, n}, N_{G_{m, n}}(\mathrm{x})$ is an $x-y$ rainbow node-cut. For any two adjacent nodes $\mathrm{x}$ and y of $G_{m, n}, N_{G_{m, n}}(\mathrm{x}) \backslash\{\mathrm{y}\}$ is an $x-y$ rainbow node-cut. The coloring c is a rainbow node-disconnection coloring of $G_{m, n}$, . Hence, $\operatorname{rnd}(\mathrm{G}) \leq 4$. On the other hand, $\kappa\left(x_{2,2}, x_{3,3}\right)=4$. It follows by Lemma 3.3 that $\operatorname{rnd}\left(G_{m, n},\right) \geq \mathrm{\kappa}\left(x_{2,2}, x_{3,3}\right)=4$.

For a node-cut $S$ of $G$, we denote the connected components of $G-S$ by $G_{1}, G_{2}, \cdots, G_{s}$. Then we add $S$ to these components and get networks $G\left[V\left(G_{1}\right)+S\right], G\left[V\left(G_{2}\right)+S\right], \cdots, G\left[V\left(G_{s}\right)+S\right]$. This operation is called that we split the node-cut $S$.

If the nodes of a 2-node-cut of $G$ are adjacent, then we say the 2-node-cut is an adjacent 2-node-cut.

Theorem 3.7 Let $G$ be a grid network. Then (as shown in Figure 4)

$$
\operatorname{rnd}(G)= \begin{cases}2, & \text { if } G=G_{1}, \\ 3, & \text { if } G_{2} \subseteq G \text { and } G_{3}, G_{4} \nsubseteq G, \\ 4, & \text { if } G_{3} \subseteq G \text { or } G_{4} \subseteq G .\end{cases}
$$




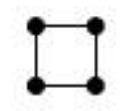

$G_{1}$

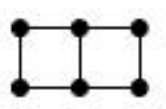

$G_{2}$

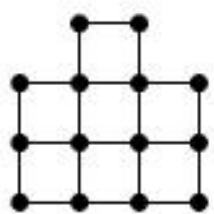

$G_{3}$

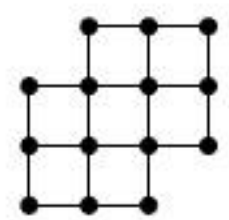

$G_{4}$

Figure 4: Grid networks in Theorem 3.7.

Proof. If $G=G_{1}$, then we have $\operatorname{rnd}(G)=2$ by Lemma 3.1. If $G_{3} \subseteq G$ or $G_{4} \subseteq G$, then $\operatorname{rnd}(G) \geq \kappa^{+}(G) \geq$ 4 by Lemma 3.3. Since $G$ is the subnetwork of some grid network $G_{m, n}$, we have $\operatorname{rnd}(G) \leq \operatorname{rnd}\left(G_{m, n}\right)=$ 4 by Lemmas 3.2 and 3.6.

Now consider $G_{2} \subseteq G$ and $G_{3}, G_{4} \not \subseteq G$.

We have $\operatorname{rnd}(G) \geq \kappa^{+}(G) \geq \kappa^{+}\left(G_{2}\right) \geq 3$. If $G=G_{3, n}$, then $\operatorname{rnd}(G)=3$ by Lemma 3.5.

If $G \neq G_{3, n}$, then there exists an adjacent 2-node-cut. We split all adjacent 2-node-cuts. Then we can get networks $H_{1}, H_{2}, \cdots, H_{\ell}$. Obviously, each $H_{i}$ is a 4-cycle or $G_{3, n}$. Then we do the following operations.

1. Select the network $H_{1}$ and color $H_{1}$ using rnd-coloring $c_{1}$. Let $H=H_{1}$ and $c_{H}=c_{1}$.

2. Select the network $H_{i}$ which has a common adjacent 2-node-cut $S$ with network $H$ and color $H_{i}$ using rnd-coloring $c_{i}$.

3. Let $H=H \cup H_{i}$ and $c_{H}=c_{H}+c_{i}$. If $H$ and $G$ are not isomorphic, then return to step 2.

The rnd-colorings $c_{i}(i \in[\ell])$ are as follows.

$c_{1}$ : If $H_{1}$ is a 4-cycle, then we assign color 1 to two adjacent nodes and assign 2,3 to the remaining two nodes. If $H_{1}$ is $G_{3, n}$, then we color it using the same coloring as Lemma 3.5.

$c_{i}(i \in\{2,3, \cdots, \ell\})$ : Assume that $S=\{u, v\}$. Let $c_{i}(u)=c_{H}(u)$ and $c_{i}(v)=c_{H}(v)$.

If $H_{i}$ is a 4-cycle, we denote the 4-cycle containing link $u v$ in $H$ by $C_{i}$. We color the neighbors of $u$ and $v$ in $H_{i}$ using the colors different from $N_{C_{i}}(u)$ and $N_{C_{i}}(\mathrm{v})$ respectively. Obviously, we finish the color of $H_{i}$.

Next, consider $H_{i}=G_{3, n}$. Obviously, $u, v$ have at least one node with degree four in $G$ and degree two in $H$. Without loss of generality, assume that $d_{G}(v)=4$ and $d_{H}(v)=2$. Let $N_{H}(v)=\left\{u, v_{1}\right\}$. We use two stages to color $H_{i}$.

- If $d_{G}(u)=3$, then color the neighbor of $u$ in $H_{i}$ such that $N_{G}(u)$ is rainbow.

If $d_{G}(\mathrm{u})=4$, then $d_{H_{i}}(u)=2$. Let $N_{H_{i}}(u)=\left\{v, u_{1}\right\}$. When $\left\{u, v, v_{1}\right\}$ is rainbow, let $c_{i}\left(u_{1}\right)=c_{i}(u)$; otherwise, color $u_{1}$ such that $\left\{u_{1}, v_{1}, u\right\}$ is rainbow.

- Color the remaining nodes of $H_{i}$ according to Figure 5. 
In first stage, we color three nodes of $H_{i}$. No matter how we color it, the colors of three nodes have three cases as shown in Figure 5, where the three nodes are marked by stars and $\{a, b, c\}=\{1,2,3\}$ are three different colors.

In second stage, for the networks in Figure 5, other columns of $H^{\prime}, H^{\prime \prime}$ and $H^{\prime \prime}$ repeat the colors of columns 1-4.

Similar to the proof of Lemma 3.5, we can get that $c_{i}$ is an rnd-coloring of $H_{i}$ for $i \in[\ell]$.

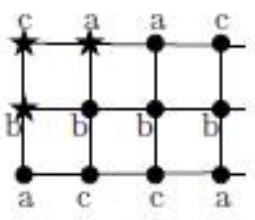

$H^{\prime}$

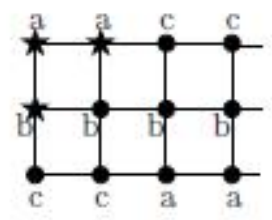

$H^{\prime \prime}$

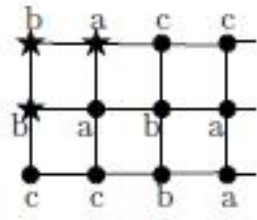

$H^{\prime \prime \prime}$

Figure 5: Three node-colorings of $G_{3, n}$.

Now we claim that the node-coloring of $H \cup H_{i}$ is an rnd-coloring. Based on the process of coloring, the neighborhoods of nodes with degree less than four are rainbow. So we only need to consider two nodes with degree four.

Let $x, y$ be two nodes of $H \cup H_{i}$ with degree four. Assume that $R_{H}$ is an $x-y$ rainbow node-cut of $H$ under $c_{H}$. Let $R_{i}$ be an $x-y$ rainbow node-cut of $H_{i}$ under $c_{i}$. Consider $\{x, y\}=\{u, v\}$. Then $\left\{u_{1}, v 1, v\right\}$ or $\left\{u_{1}, v_{1}, u\right\}$ is an $x-y$ rainbow node-cut of $H \cup H_{i}$.

Consider $\{x, y\} \neq\{u, v\}$. If $x, y \in V(H)$, then $R_{H}$ is an $x-y$ rainbow node-cut of $H \cup H_{i}$. If $x, y \in V\left(H_{i}\right)$, then $R_{i}$ is an $x-y$ rainbow node-cut of $H \cup H_{i}$. If $x \in V(H) \backslash\{u, v\}, y \in V\left(H_{i}\right) \backslash\{u, v\}$ or $x \in V\left(H_{i}\right) \backslash$ $\{u, v\}, y \in V(H) \backslash\{u, v\}$, then $\left\{v, u_{1}\right\}$ is an $x$-y rainbow node-cut of $H \cup H_{i}$.

So the above operations keep new network $H=H \cup H_{i}$ rainbow node-disconnected. Therefore, $\operatorname{rnd}(G)$ $=3$.

\section{THEIR PD-NUMBERS AND MD-NUMBERS}

Furthermore, we obtain the proper and monochromatic disconnection numbers of cellular networks and grid networks.

Observation 4.1 Let $G$ be a cellular network. Then $p d(G)=1$.

Observation 4.2 Let $G$ be a grid network. Then $p d(G)=1$.

Theorem 4.3 Let $G$ be a cellular network with the number of hexagons $h$, the number of inner links $m$ and the boundary $C$. Then $m d(G)=3 h-m=|C| / 2$.

Proof. Observe that each color appears at least 2 times in an md-coloring, so one hexagon has at most 3 colors. If two hexagons have a common link, then the two hexagon use at most 5 colors under an md-coloring in $G$. Then an md-coloring of $G$ has at most $3 h-m$ colors since $G$ has $m$ pairs of hexagons with a common link. Namely, $\operatorname{md}(G) \leq 3 h-m$. 
Now we give a coloring $f$ of $G$. First, we give a link partition for $G$. For two adjacent hexagons $H_{1}, H_{2}$, let $e$ be the common link of $H_{1}, H_{2}$. Then there are opposite links $e_{1}$ and $e_{2}$ of $e$ in $H_{1}$ and $H_{2}$, respectively. If $e_{1}$ or $e_{2}$ is not a bounded link, then we continue to find the opposite link of $e_{1}$ or $e_{2}$ in other hexagon, and call all these opposite links a relative link set, denoted by $M_{i},(i \in[t])$. Observe that $\mathrm{E}(\mathrm{G})=\mathrm{U}_{i=1}^{t} M_{i}$ and $t=|C| / 2$. Next, for each $i \in[t]$, we assign color $i$ to all links of $M_{i}$, therefore $|f|=t$. Moreover, we get $|f|=3 h-m$ since $|C|+m=6 h-m$. It is easy to verify that the coloring $f$ is an md-coloring of $G$. Hence, $\operatorname{md}(G)=3 h-m$.

Theorem 4.4 Let $G$ be a grid network with the number of quadrangle $h$, the number of inner links $m$ and the boundary $C$. Then $m d(G)=2 h-m=|C| / 2$.

The proof of Theorem 4.4 is similar to the argument of Theorem 4.3.

\section{Conclusions}

In this paper, we get the exact values of the rainbow (node-)disconnection numbers, proper and monochromatic disconnection numbers of cellular networks and grid networks, respectively, and we conjecture that the strong rainbow disconnection numbers of grid networks are equal to the rainbow disconnection numbers of grid networks.

\section{ACKNOWLLINKMENTS}

The authors are very grateful to the reviewers and editor for their useful suggestions and comments, which helped to improve the presentation of the paper. This work was supported by NSFC No.11871034. X. Li is the corresponding author.

\section{REFERENCES}

[1] W. A11 cock, J. Bester, J. Bresnahan, A. Chervenal, I. Foster, C. Kesselman, S. Meder., V. Nefedova, D. Quesnel \& S.Tuecke, (2001) "Data managememt and transfer in high-performance computational grid envimnments", Parallel Computing.

[2] W. Allcock, J. Bresnahan, I. Foster, L Liming, L Link \& P. Plaszczac, (2002) Tecnical Report: GridFTP Update. January.

[3] X. Bai, Y. Chen, P. Li, X. Li \& Y. Weng, (2021) "The rainbow vertex-disconnection in graphs", Acta Mathematica Sinica, English Series, Vol. 37, No. 2, pp249-261.

[4] X. Bai, Z. Huang \& X. Li, "Bounds for the rainbow disconnection number of graphs", Acta Mathematica Sinica, English Series accepted for publication. arXiv:2003.13237.

[5] X. Bai \& X. Li, (2021) "Strong rainbow disconnection in graphs", International Symposium on Automation, Information and Computing, J. Phys.: Conf. Ser. 1828, 012150.

[6] X. Bai \& X. Li, "Graph colorings under global structural conditions", arXiv:2008.07163, manuscript.

[7] J.A. Bondy \& U.S.R. Murty (2008) Graph Theory, Graduate Texts in Mathematics 244, Springer.

[8] G. Chartrand, S. Devereaux, T.W. Haynes, S.T. Hedetniemi \& P. Zhang, (2018) "Rainbow disconnection in graphs", Discuss. Math. Graph Theory, Vol. 38, No. 4, pp1007-1021.

[9] I. Foster, Internet Computing and the emerging grid, (2000) Nature web matters, http://www.nature.com/nature/webmatters/grid/grid.html,

[10] G. Chartrand, G.L. Johns, K.A. McKeon \& P. Zhang, (2008) "Rainbow connection in graphs", Math. Bohem., Vol. 133, pp85-98.

[11] I. Gutman, (1982) “Topological properties of hexagonal molecules", Bull. Soc. Chim. Beograd, Vol. 47, pp453.

[12] M. Krivelevich \& R. Yuster, (2010) "The rainbow connection of a graph is (at most) reciprocal to its minimum degree", J. Graph Theory, Vol. 63, pp185-191. 
[13] X. Li \& Y. Shi, (2013) “On the Rainbow vertex-connection”, Discuss. Math. Graph Theory, Vol. 33, pp307-313.

[14] X. Li \& Y. Shi, Y. Sun, (2013) "Rainbow connections of graphs: A survey", Graphs Combin., Vol. 29, pp1-38.

[15] X. Li \& Y. Sun (2012) Rainbow Connections of Graphs, New York, Springer Briefs in Math., Springer.

[16] X. Li \& Y. Sun, (2017) "An updated survey on rainbow connections of graphs - a dynamic survey", Theory Appl. Graphs., Vol. 0, Art. 3.

[17] P.G. Tait, (1880) "Remarks on coloring of maps", Proc. Roy. Soc. Edinburgh Sect. A, Vol. 10, pp729.

[18] J. Epstein (2009) Chapter 7-Voice over Cellular and Licensed Spectrum - ScienceDirect., Scalable VoIP Mobility, Newnes, pp289-322.

[19] D.T. Ngo, D.H.N. Nguyen \& T. Le-Ngoc (2013) Chapter 6-Intercell Interference Coordination: Towards a Greener Cellular Network, Handbook of Green Information and Communication Systems, Academic Press, pp147-182.

(C) 2021 By AIRCC Publishing Corporation. This article is published under the Creative Commons Attribution (CC BY) license. 